\title{
COMPLEX CONTACT MANIFOLDS AND HYPERKÄHLER GEOMETRY*
}

\author{
BRENDAN FOREMAN ${ }^{\dagger}$
}

\begin{abstract}
We investigate the properties of a certain class of hermitian metrics on complex contact manifolds with global complex contact forms. These are called complex Sasakian metrics. In the main theorem, we prove a relationship between complex Sasakıan geometry and hyperkähler geometry. We then compare these complex contact manifolds to the more famous twistor space examples.
\end{abstract}

\section{Introduction}

In times past, there have been many interesting and useful correspondences proven between real contact and almost complex geometries. For example, Hatakeyama proved in [12] that every Sasakian manifold locally fibres over a Kähler manifold. Also, the Boothby-Wang Fibration gives a construction by which an $S^{1}$-bundle with a real contact structure is formed over a symplectic manifold [5]. Furthermore, Hatakeyama's result can be generalized to make the Boothby-Wang fibration a Riemannian submersion of associated metrics.

A great deal work has also been done to find similar correspondences dealing with complex contact and quaternionic structures on manifolds. However, a large obstacle has been finding a proper quaternionic context to work with. An almost quaternionic structure is defined easily enough; it is simply a metric $g$ together with a three-dimensional bundle $E$ of endomorphisms with local bases $\{A, B, C=A B\}$ of local anti-commuting almost complex structures such that $g$ is hermitian with respect to each endomorphism.

What is the analogue for $g$ being Kähler? If we just insist that each almost complex structure of $E$ be parallel, then the quaternionic projective space $H P^{n}$ would be excluded (see [3]). To remedy this, we define an almost quaternionic manifold $(M, g, E)$ to be quaternionic-Kähler, if $\nabla E \subset T^{*} M \otimes E$. Under these circumstances, $g$ is Einstein, and so we can categorize these manifolds into three categories by the sign of the scalar curvature: zero, positive, or negative.

\footnotetext{
*1991 Mathematics Subject Classification. Primary 53T58.

${ }^{\dagger}$ Research supported by NSF grant \#DMS95-20481

Received October 1, 1998; revised December 15, 1999.
} 

setting

For any of these cases, we can create the twistor space $Z$ of $M$ by locally

$$
Z=\left\{x A+y B+z C: x^{2}+y^{2}+z^{2}=1\right\} \subset E .
$$

Salamon and Bérard-Bergery ([22], [2]) both showed that, if the scalar curvature of $g$ is nonzero, then $Z$ carries both a natural complex structure and complex contact structure derived from a connection for the fibration $E \rightarrow M$. Thus, we get a correspondence between certain classes of quaternionic and complex contact manifolds.

When the scalar curvature is zero, however, this construction will not produce a complex contact manifold. In fact, $E$ will be trivial, and we will have a global set of parallel complex structures $\{A, B, C\}$. Such an almost quaternionic manifold is called hyperkähler.

In this paper, we show that this class of quaternionic manifolds does correspond with a certain class of complex contact manifolds, called strict normal complex contact manifolds. These complex contact manifolds are very different from twistor spaces, especially since they reside in the so-called "strict complex contact" category of manifolds. This category of complex contact manifolds has for the most part been ignored, even though a certain element of it, the complex Heisenberg group, is very well known.

In the first section, we introduce and describe some properties of these types of manifolds. Then we investigate their hermitian geometry. In the fourth section, we introduce the notion of a complex Sasakian metric and prove the main theorem which relates complex Sasakian metrics with hyperkähler geometry. In the fifth section, we briefly describe some examples of complex Sasakian manifolds. In the last section, we compare some aspects of complex Sasakian manifolds with the much more famous examples of complex contact manifolds, the twistor spaces of quaternionic Kähler manifolds with non-zero scalar curvature; and we prove that, like complex Sasakian manifolds, we can think of these twistor spaces as complex contact manifolds satisfying certain curvature equations.

\section{Strict complex contact manifolds}

Recall that a complex contact manifold is a complex manifold $(M, J)$ of complex dimension $2 n+1$ such that there is an open atlas $\mathscr{U}=\{\mathcal{O}\}$ of $M$ for which the following statements hold:

1. On each $\mathcal{O} \in \mathscr{U}$, there is a holomorphic 1 -form $\eta$ such $\eta \wedge(d \eta)^{n} \neq 0$ everywhere on $\mathcal{O}$.

2. For $\mathcal{O}, \mathcal{O}^{\prime} \in \mathscr{U}$ with respective 1 -forms $\eta, \eta^{\prime}$, there is holomorphic function $f: \mathcal{O} \cap \mathcal{O}^{\prime} \rightarrow \boldsymbol{C}^{*}$ such that $\eta^{\prime}=f \eta$.

If we define $\mathscr{H}^{1,0}=\left(\bigcup_{\mathcal{O} \in \mathscr{U}} \operatorname{ker}(\eta)\right) \cap T^{1,0} M$, then $\mathscr{H}^{1,0}$ is a well-defined, holomorphic subbundle of $T^{1,0} M$ of maximal rank and complex dimension $2 n$, called 
the holomorphic contact subbundle of $M$. Set $\mathscr{H}=\left\{X \in T^{R} M: X-i J X \in \mathscr{H}^{1,0}\right\}$. Then $\mathscr{H}$ is a subbundle of $T M$ with maximal rank, which is holomorphic in the sense that there are local bases of $\mathscr{H}\left\{X_{1}, \ldots, X_{4 n}\right\}$ such that $\mathrm{Ł}_{X_{l}} J=0$ for $j=1, \ldots, 4 n$. We will call $\mathscr{H}$ the holomorphic contact subbundle or simply the contact subbundle.

Let $(M, J)$ be a complex contact manifold with complex contact subbundle $\mathscr{H}$. We say that a set of $C$-valued 1 -forms $\{\pi\}$ whose domains cover $M$ is a normalized contact structure, if any two 1 -forms $\pi, \pi^{\prime}$ in this set with respective domains $\mathcal{O}, \mathcal{O}^{\prime}$ satisfy:

1. $\mathscr{H}=\operatorname{ker}(\pi)$ on $\mathcal{O}$.

2. On $\mathcal{O} \cap \mathcal{O}^{\prime}, \pi^{\prime}=f \pi$ for some $f: \mathcal{O} \cap \mathcal{O}^{\prime} \rightarrow S^{1}$.

Note that for any $\pi$ in this set, $\pi=u-i v$ where $u$ and $v$ are real 1-forms such that $v=u \circ J$. By taking a hermitian metric on the complex line bundle $L=$ $\left\{\omega \in \Lambda^{1,0} M: \omega(\mathscr{H})=0\right\}$, it is fairly easy to construct a normalized contact structure on $M$. This very construction shows that such structures are far from unique. However, they are handy objects to use because of the next theorem (see [8] for proof).

THEOREM 2.1. Given a normalized contact structure $\{\pi\}$ on a complex contact manifold $M$, there is a unique, two-dimensional, J-invariant subbundle $\mathscr{V}$ of TM such that

1. $T M \cong \mathscr{H} \oplus \mathscr{V}$.

2. For any element $\pi=u-\imath$ of the normalized contact structure, there is a local basis of $\mathscr{V},\{U, J U\}$ defined by:

(a) $u(U)=1, v(U)=0, u(J U)=0, v(J U)=-1$,

(b) $d u(U, X)=0, d v(J U, X)=0$ for any $X \in \mathscr{H}$.

Since there is a splitting $T M \cong \mathscr{H} \oplus \mathscr{V}$, we have two natural projections $T M \rightarrow \mathscr{H}$ and $T M \rightarrow \mathscr{V}$. We will denote these respective projections by the same name as their corresponding subbundles, $\mathscr{H}$ and $\mathscr{V}$.

We say $M$ is a strict complex contact manifold, if there 1 a global holomorphic 1 -form $\eta$ such that $\mathscr{H}=\operatorname{ker}(\eta)$. With such a manifold, we let $\tilde{U}$ be the unique holomorphic vector field given by the equations $\eta(\tilde{U})=1$, and $\imath(\tilde{U}) d \eta=0$. Also, the singleton set $\{\eta\}$ is a normalized contact structure on $M$. The resulting vertical subbundle $\mathscr{V}$ is given by $\mathscr{V}=\operatorname{span}_{R}\{U, J U\}$ where $U$ and $J U$ are given by $\tilde{U}=(1 / 2)(U-i J U)$. Since $\tilde{U}$ is holomorphic, both $U$ and $J U$ are infinitesimal automorphisms of $J$, i.e., $\mathrm{七}_{U} J=\mathrm{七}_{J U} J=0 . \quad$ In particular, $[U, J U]=0$ so that $\mathscr{V}$ is a foliation on $M$.

Note that, under these circumstances, $d u(U, J U)=-(1 / 2) u([U, J U])=0$. Also, $d v(U, J U)=0$. This departs sharply from the general case of complex contact manifolds (e.g., see [17]). 


\section{Hermitian geometry on strict complex contact manifolds}

We now examine some aspects of the hermitian geometry of strict complex contact manifolds. To this end, we will restrict ourselves to hermitian metrics which exploit the complex contact structure on the given manifold. From this point on, we will assume that $(M, J, \eta)$ is a compact strict complex contact manifold with global complex contact structure $\eta$. We will use the same notation as the previous section. In particular, $\tilde{U}=(1 / 2)(U-i J U)$ is the holomorphic vertical vector field defined as before by $\eta$.

A hermitian metric $g$ on $(M, J, \eta)$ is called associated to $\eta$, if it satisfies the following statements:

1. The real endomorphisms $G, H$ defined by

$$
\begin{aligned}
& G(X)=-\operatorname{skew}\left(X \mapsto \nabla_{X} U\right), \\
& H(X)=\operatorname{skew}\left(X \mapsto \nabla_{X}(J U)\right)
\end{aligned}
$$

satisfy $G^{2}=H^{2}=-i d_{\mathscr{H}}, H=G J=-J G, G \mathscr{V}=0$.

2. $\eta(X)=g(X, U)+i g(X, J U)$.

Thus, the covariant derivatives $\nabla U$ and $\nabla(J U)$ of an associated metric induce a quaternionic structure $\left\{G, H, J^{\prime}=J \circ \mathscr{H}\right\}$ on $\mathscr{H}$. Associated metrics were shown to exist in [17]. In fact, the family of associated metrics forms an infinitedimensional manifold (see [8]). Note that an associated metric on a general complex contact manifold is simply defined by saying that the local endomorphisms $G$ and $H$ given above by a normalized contact structure $\{\pi\}$ form a local quaternionic structure on $\mathscr{H}$.

If we set $\eta=u-v$, then $d u(X, Y)=g(X, G Y)$ and $d v(X, Y)=g(X, H Y)$. Also, $u(X)=g(X, U)$ and $v(X)=-g(X, J U)$.

Since $\mathscr{V}$ is a foliation, we may locally fibre out the vertical leaves of $M$, $\pi_{\mathscr{C}}: \mathcal{O} \rightarrow \tilde{\mathcal{O}}=\mathcal{O} / \mathscr{V}$. We say that an associated metric $g$ is projectable, if, for each such fibration, there is a metric $\tilde{g}$ on $\tilde{\mathcal{O}}$ such that $g_{\mathscr{H}}=\left(\pi_{\mathcal{C}}\right)^{*} \tilde{g}$. It is known ([15]) that this is equivalent to the condition $\left(\mathrm{L}_{W}(g \circ \mathscr{H})\right)_{\mathscr{H}}=0$ for any vertical vector field $W$.

We define real endomorphisms $h_{U}, h_{J U}$ by $h_{U}(X)=\operatorname{sym}\left(X \mapsto \nabla_{X} U\right)$ and $h_{J U}(X)=\operatorname{sym}\left(X \mapsto \nabla_{X} J U\right)$. Then $\nabla_{X} U=-G X+h_{U}(X), \quad$ and $\nabla_{X}(J U)=$ $H X+h_{J U} X$. It can be shown that $G \circ h_{U}=-h_{U} \circ G$ and $H \circ h_{J U}=-h_{J U} \circ H$, however the proof is far too complicated for this paper. See [8] for the full proof.

These endormorphisms have a nice geometric interpretation. It is easily seen that for any vertical vector field $W=a U+b J U, X, Y \in \mathscr{H},\left(\mathfrak{七}_{W} g\right)(X, Y)=$ $a g\left(h_{U}(X), Y\right)+b g\left(h_{J U}(X), Y\right)$. So, we see that $g$ is projectable if and only if $h_{U}=h_{J U}=0$. This shows that $W$ is Killing. Thus, a projectable associated metric is the complex analogue of a $K$-contact metric on a real contact manifold. To this effect and for future reference, we mention one more projectability equivalence. 
Proposition 3.1. An associated metric $g$ on a strict complex contact manifold is projectable if and only if the sectional curvature of any non-vertical plane section containing an element of $\mathscr{V}$ is equal to 1.

We now prove a few identities of associated metrics.

LEMMA 3.2. $\nabla_{U} G=\nabla_{J U} H=0$.

Proof. Since $d u(X, Y)=g(X, G Y)$, we know $0=g\left(\left(\nabla_{X} G\right) Y, Z\right)+$ $g\left(\left(\nabla_{Y} G\right) Z, X\right)+g\left(\left(\nabla_{Z} G\right) X, Y\right)$. Substituting ' $U$ ' for ' $X$ ' and using the skewsymmetry of $\nabla G$, we see that $g\left(\left(\nabla_{U} G\right) Y, Z\right)=g\left(\left(\nabla_{Y} G\right) U, Z\right)-g\left(\left(\nabla_{Z} G\right) U, Y\right)$. However, $\left(\nabla_{Y} G\right) U=-G\left(\nabla_{Y} U\right)=-\mathscr{H} Y+h_{U} Y$. Since both $\mathscr{H}$ and $h_{U}$ are symmetric operators, $\nabla_{U} G=0$. The proof for $\nabla_{J U} H=0$ is similar.

LEMMA 3.3. $\nabla_{U} J=-2 H, h_{U} \circ J=J \circ h_{U}$.

Proof. Since $U$ is an infinitesimal automorphism of $J$,

$$
\begin{aligned}
0 & =[U, J X]-J[U, X] \\
& =\nabla_{U}(J X)-\nabla_{J X} U-J \nabla_{U} X+J \nabla_{X} U \\
& =\left(\nabla_{U} J\right) X+2 H X-h_{U} J X+J h_{U} X .
\end{aligned}
$$

However, the operator $\left(\nabla_{U} J+2 H\right)$ is skew-symmetric with respect to $g$, whereas $\left(-h_{U} J+J h_{U}\right)$ is symmetric. Thus, $0=\nabla_{U} J+2 H$, and $0=h_{U} J X-J h_{U} X$.

LEMMA 3.4. $g\left(R_{X Y} U, Z\right)=g\left(\left(\nabla_{Z} G\right) X, Y\right)+g\left(\left(\nabla_{X} h_{U}\right) Y, Z\right)-g\left(\left(\nabla_{Y} h_{U}\right) X, Z\right)$.

Proof. This follows from carrying $R_{X Y} U$ out by using the definitions of $G$ and $h_{U}$ then using the fact that the 2-form $\hat{G}(X, Y)=g(X, G Y)$ is closed.

\section{Complex Sasakian metrics}

For a nice exposition of the upcoming propositions, we need some notation. Let $V$ be a vector space over $\boldsymbol{R}$. For a 1-form $\alpha: V \rightarrow \boldsymbol{R}$ and endomorphism $\beta: V \rightarrow V$, we let $\alpha \wedge \beta$ be the rather obvious skew-symmeric, bilinear function $V \times V \rightarrow V$ given by $(\alpha \wedge \beta)(X, Y)=(1 / 2)(\alpha(X) \beta(Y)-\alpha(Y) \beta(X))$.

Proposition 4.1. Suppose that $g$ is an associated metric for a strict complex contact manifold $(M, J, \eta)$. Then the following statements are equivalent. $X, Y$.

1. $R_{X Y} U=-2(u \wedge \mathscr{H})(X, Y)+2\left(v \wedge J^{\prime}\right)(X, Y)+2 g\left(J^{\prime} X, Y\right) V$ for any

2. $\left(\nabla_{X} G\right) Y=g(\mathscr{H} X, Y) U+g\left(J^{\prime} X, Y\right) V-u(Y) \mathscr{H} X-v(Y) J^{\prime} X-2 v(X) J^{\prime} Y$ for any $X, Y$. 
Proof. It is easily seen that, by Lemma (3.4), the statements are equivalent, provided that $h_{U}=0$. Thus, we need only to show that for both statements $h_{U}=0$.

If 1. is true, then, for $X \in \mathscr{H}, R_{U X} U=-X$. By Proposition (3.1), we see that $g$ is projectable. For any associated metric $g,\left(\nabla_{X} G\right) U=-\mathscr{H} X-G h_{U} X$ for all $X$. If 2 . is true, then we also have $\left(\nabla_{X} G\right) U=-\mathscr{H} X$. Thus, $h_{U}=0$. This proves the proposition.

There is an analogous proposition concerning $J U$ and $H$ which we now give.

Proposition 4.2. Suppose that $g$ is an associated metric for a strict complex contact manifold $(M, J, \eta)$. Then the following statements are equivalent. $X, Y$.

1. $R_{X Y} J U=2(v \wedge \mathscr{H})(X, Y)+2\left(u \wedge J^{\prime}\right)(X, Y)+2 g\left(J^{\prime} X, Y\right) U$ for any

2. $\left(\nabla_{X} H\right) Y=g\left(J^{\prime} X, Y\right) U-g(\mathscr{H} X, Y) V+u(Y) J^{\prime} X-v(Y) \mathscr{H} X-2 u(X) J^{\prime} Y$ for any $X, Y$.

An associated metric satisfying one statement from each of the two propositions above is called complex Sasakian. This definition is a special case of the so-called normal complex contact metrics which is due to B. Korkmaz (nee Karaman) in [20]. The original definition involves associated metrics on any complex contact manifold, not just strict ones. Thus, it is a great deal more complicated. In particular, twistor spaces over quaternionic Kähler manifolds with positive scalar curvature have normal complex contact metrics-namely the so-called Salamon-Bérard-Bergery metrics. Results about normality and twistor spaces will be forthcoming in [10].

Going back to the strict category, the above proof and the analogous proof for Proposition (4.2) gives us this proposition.

Proposition 4.3. Every complex Sasakian metric is projectable.

We now go to the main theorem of this paper.

THEOREM 4.4. Let $(M, J, \eta)$ be a complex contact manifold with global structure. Let $g$ be an associated metric with horizontal endomorphisms $\left\{G, H, J^{\prime}\right\}$. Then $g$ is complex-Sasakian if and only if $g$ is projectable and "projects" locally to a hyperkähler manifold with hypercomplex structure corresponding to $\left\{G, H, J^{\prime}\right\}$.

Proof. We know that $g$ is projectable (i.e., $h=0$ ). Also, $g$ satisfies:

$\left(\nabla_{Z} G\right) X=g(\mathscr{H} X, Z) U-g\left(J^{\prime} X, Z\right) V-u(X) \mathscr{H} Z-v(X) J^{\prime} Z-2 v(Z) J^{\prime} X$,

$\left(\nabla_{Z} H\right) X=g\left(J^{\prime} X, Z\right) U-g(\mathscr{H} X, Z) V+u(X) J^{\prime} Z-v(X) \mathscr{H} Z-2 u(Z) J^{\prime} X$,

$\left(\nabla_{Z} J\right) X=-2 u(Z) H X+2 v(Z) G X$.

In particular, $\mathscr{H}\left(\nabla_{X} G\right) Y=\mathscr{H}\left(\nabla_{X} J\right) Y=\mathscr{H}\left(\nabla_{X} H\right) Y=0$ for any horizontal $X, Y$. 
Locally, since $\mathscr{V}$ is a foliation, we can fibre by vertical leaves: $\pi: \mathcal{O} \rightarrow \tilde{\mathcal{O}}=$ $\mathcal{O} / \mathscr{V}$. Since $g$ is projectable, there is a metric $\tilde{g}$ on $\mathcal{O} / \mathscr{V}$ such that $g=\pi^{*}(\tilde{g})+$ $u \otimes u+v \otimes v$. Let $\nabla$ and $\tilde{\nabla}$ be the respective Levi-Civita connections of $g$ and $\tilde{g}$.

We know $U+i V$ is a holomorphic vector field on $M$ so that $\mathrm{七}_{U} J=$ $\mathrm{L}_{V} J=0$. So $J$ is also projectable. This means that there is an almost complex structure $\tilde{J}$ on $\tilde{\mathcal{O}}$ such that $\pi \circ J=\tilde{J} \circ \pi$. It is easily seen that $\tilde{J}$ is integrable (since $J$ is), and so $\pi$ is a holomorphic submersion of open complex manifolds.

Using the fact that $\nabla_{U} G=\nabla_{V} H=0$, we see that $\mathrm{七}_{U} G=\mathrm{七}_{V} H=0$. Also, $\mathrm{Ł}_{U} H=\mathrm{Ł}_{U}(G J)=\left(\mathrm{Ł}_{U} G\right) J+G\left(\mathrm{Ł}_{U} J\right)=0$. Similarly, $\mathfrak{七}_{V} G=0$. Hence, both $G$ and $H$ are projectable endomorphisms, corresponding to endomorphisms $\tilde{G}$ and $\tilde{H}$ on $\mathcal{O} / \mathscr{V}$. Since $(\pi)_{*}$ restricted to $\mathscr{H}$ is an isomorphism onto $T \mathcal{O} / \mathscr{V}$, we see that $\tilde{G}$ and $\tilde{H}$ are almost complex structures on $\mathcal{O} / \mathscr{V}$. In particular, $\{\tilde{G}, \tilde{J}, \tilde{H}\}$ form an almost quaternionic structure on $\mathcal{O} / \mathscr{V}$.

Let $m \in \mathcal{O}$ with $\tilde{m}=\pi(m)$. Also, $X$ and $Y$ be basic vector fields on $\mathcal{O}$ with $\pi_{*}(X)=\tilde{X}$ and $\pi_{*}(Y)=\tilde{Y}$. Note that since $X$ and $G$ are projectable tensors on $\mathcal{O}, \pi_{*}(G X)=\tilde{G}\left(\pi_{*}(X)\right)=\tilde{G} \tilde{X}$. Similar identities exist for $H$ and $J$. We denote by hor $_{m}: T_{\tilde{m}} \tilde{\mathcal{O}} \rightarrow T_{m} \mathcal{O}$ the horizontal lift map on $T_{\tilde{m}} \tilde{\mathcal{O}}$. Then we have

$$
\left(\nabla_{X} Y\right)_{m}=\operatorname{hor}_{\tilde{m}}\left(\tilde{\nabla}_{\tilde{X}} \tilde{Y}\right)+\frac{1}{2} \mathscr{V}\left([X, Y]_{m}\right) .
$$

In particular, we find that $\left(\tilde{\nabla}_{\tilde{X}} \tilde{G}\right) \tilde{Y}=\pi_{*}\left(\left(\nabla_{X} G\right) Y\right)=\pi_{*}\left(\mathscr{H}\left(\left(\nabla_{X} G\right) Y\right)\right)=0$. Similarly, we find that $\tilde{J}$ and $\tilde{H}$ are also parallel with respect to $\tilde{g}$. Thus, $\{\mathcal{O} / \mathscr{V}, \tilde{g},\{\tilde{G}, \tilde{H}, \tilde{J}\}\}$ is a hyperkähler manifold.

Conversely, let us now suppose that we have a local Riemannian submersion $(\mathcal{O}, g) \rightarrow(\mathcal{O} / \mathscr{V}, \tilde{g})$ such that $\{G, H, J\}$ on $\mathcal{O}$ projects to $\tilde{g}$-parallel hypercomplex structure $\{\tilde{G}, \tilde{H}, \tilde{J}\}$. Let $m \in \mathcal{O}$ with $\tilde{m}=\pi(m), X, Y \in \mathscr{H}$ be basic vector fields defined at $m$ with corresponding vector fields $\tilde{X}, \tilde{Y}$ on $\mathcal{O} / \mathscr{V}$. Then

$$
\begin{aligned}
\left(\nabla_{X} G\right) Y & =\nabla_{X}(G Y)-G\left(\nabla_{X} Y\right) \\
& =\operatorname{hor}_{\tilde{m}}\left(\tilde{\nabla}_{\tilde{X}}(\tilde{G} \tilde{Y})\right)+\frac{1}{2} \mathscr{V}([X, G Y])-\operatorname{hor}_{\tilde{m}}\left(\tilde{G} \tilde{\nabla}_{\tilde{X}} \tilde{Y}\right) \\
& =\frac{1}{2} u([X, G Y]) U+\frac{1}{2} v([X, G Y]) V \\
& =-g\left(X, G^{2} Y\right) U-g(X, H G Y) V \\
& =g(X, Y) U-g\left(X, J^{\prime} Y\right) V
\end{aligned}
$$

Also $\left(\nabla_{X} G\right) U=-G\left(\nabla_{X} U\right)=-X$ and $\left(\nabla_{X} G\right) V=-J^{\prime} X$. It is known already that $\nabla_{U} G=0$. Finally, using the fact that $\left(\nabla_{U} J\right)=-2 H$, we see that $\nabla_{V} G=$ $-2 J^{\prime}$. Thus,

$$
\left(\nabla_{X} G\right) Z=-2 v(X) J^{\prime} Z+g(X, \mathscr{H} Z) U-g\left(X, J^{\prime} Y\right) V-u(Z) \mathscr{H} X-v(Z) J^{\prime} X
$$

for any $X, Y \in T \mathcal{O}$. Similarly, we get the formulae for $\nabla H$ and $\nabla J$. Thus, $g$ is a complex Sasakian metric on $M$. 


\section{Examples}

The most famous complex Sasakian manifold is the complex Heisenberg group defined by

$$
H_{\boldsymbol{C}}=\left\{\left(\begin{array}{ccc}
1 & z_{1} & z_{2} \\
0 & 1 & z_{3} \\
0 & 0 & 1
\end{array}\right): z_{1}, z_{2}, z_{3} \in \boldsymbol{C}\right\} .
$$

$H_{C}$ has a global right-invariant complex contact form given by $\eta=d z_{1}-z_{2} d z_{3}$.

Furthermore, the map $\pi:\left(z_{1}, z_{2}, z_{3}\right) \mapsto\left(z_{2}, z_{3}\right)$ gives us a holomorphic fibration

$$
C \rightarrow H_{C} \rightarrow C^{2}
$$

Set $\mathscr{V}=\operatorname{ker}\left(\pi_{*}\right)$, the vertical foliation of this fibration. Also, $\eta$ defines a connection on the resulting $\boldsymbol{C}$-bundle, and we set $\mathscr{H}=\operatorname{ker}(\eta)$, the horizontal subbundle of the connection $\eta$.

If we set $\Gamma$ to be the subgroup of $H_{C}$ consisting of all elements of $H_{C}$ with Gaussian integer entries, the 3-dimensional manifold $H_{C} / \Gamma$ is called the Iwasawa manifold. This manifold is often cited as a good example of a compact complex manifold with no Kähler structure (see [11] or [7] for details).

Now, the Euclidean metric $\langle$,$\rangle on C^{2}$ is actually hyperkähler with a quaternionic structure $\{\tilde{J}, \tilde{G}, \tilde{H}\}$ given (as endomorphisms of $\boldsymbol{R}^{4}$ ) by:

$\tilde{J}=\left(\begin{array}{cc|cc}0 & -1 & 0 & 0 \\ 1 & 0 & 0 & 0 \\ \hline 0 & 0 & 0 & -1 \\ 0 & 0 & 1 & 0\end{array}\right), \quad \tilde{G}=\left(\begin{array}{cc|cc}0 & 0 & -1 & 0 \\ 0 & 0 & 0 & 1 \\ \hline 1 & 0 & 0 & 0 \\ 0 & -1 & 0 & 0\end{array}\right), \quad \tilde{H}=\left(\begin{array}{cc|cc}0 & 0 & 0 & 1 \\ 0 & 0 & 1 & 0 \\ \hline 0 & -1 & 0 & 0 \\ -1 & 0 & 0 & 0\end{array}\right)$.

Then, on $\boldsymbol{C}^{2}$,

$$
-d z_{1} \wedge d z_{2}(\tilde{X}, \tilde{Y})=\langle\tilde{X}, \tilde{G} \tilde{Y}\rangle+i\langle\tilde{X}, \tilde{H} \tilde{Y}\rangle \text { for } X, Y \in T^{R} C^{2}
$$

We can then induce a metric $g$ on $H_{C}$ which projects down via $\pi_{*}$ to $\langle$, on $C^{2}$, i.e., $g(X, Y)=\left\langle\pi_{*} X, \pi_{*} Y\right\rangle$ for $X, Y \in T H_{C}$. We do this by insisting $g(\mathscr{H}, \mathscr{V})=0$, setting $g$ on $\mathscr{H}$ to be the horizontal lift of $\langle$,$\rangle on C^{2}$, and finally defining the real and complex components of the global vector field $\partial / \partial z_{1} \in T H_{C}$ to be unit, orthogonal vectors. The metric $g$ then is a hermitian metric on $H_{C}$.

Similarly, we induce endomorphisms $G$ and $H$ on $H_{C}$ which satisfy

$$
\begin{gathered}
\tilde{G} \circ \pi_{*}=\pi_{*} \circ G, \quad \tilde{H} \circ \pi_{*}=\pi_{*} \circ H, \\
G_{\mid \mathscr{V}}=0, \quad H_{\mid \mathscr{V}}=0 .
\end{gathered}
$$

Then, it is easily seen that the structure $(g, J, G, H)$ is associated with respect to the contact form $\eta=d z_{1}-z_{2} d z_{3}$ on $H_{C}$, i.e.,

$$
d \eta(X, Y)=g(X, G Y)+i g(X, H Y) .
$$

By our main theorem, we know then that this associated metric is complex Sasakian. 
To get a compact example, we set $\Gamma^{\prime}$ to be the additive subgroup of $C^{2}$ consisting of all elements with Gaussian integer entries. Then we get the following commutative diagram of hermitian manifolds:

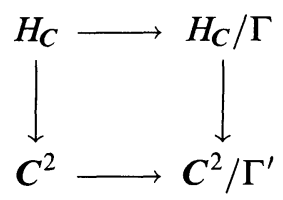

The metrics and forms of the left-hand side fibration are all left-invariant and so project over to the right-hand side fibration. Thus, the resulting metrics on $H_{C} / \Gamma$ and $C^{2} / \Gamma^{\prime}$ are complex Sasakian and hyperkähler, respectively.

It is shown in [9] that this example can be generalized to every possible dimension in a way which we'll now briefly describe. Recall that a period matrix of $\boldsymbol{C}^{N}$ is an element $W \in M_{N \times 2 N}(\boldsymbol{C})$ consisting of $n$ column vectors which are linearly independent over $\boldsymbol{R}$. Then $W Z^{2 N}$ is an additive subgroup of $\boldsymbol{C}^{N}$, and the complex manifold $C^{N} / W Z^{2 N}$ is an $N$-dimensional complex torus.

We set

$$
\tilde{J}_{2}=\left(\begin{array}{cc}
0 & -I_{n} \\
I_{n} & 0
\end{array}\right) \text {. }
$$

We call a period matrix $W$ of $C^{2 n}$ (i.e., $N=2 n$ ) a hyperkähler Riemann matrix, if there is a Hermitian matrix $G \in M_{2 n \times 2 n}(C)$ such that

1. $G>0$,

2. $\tilde{J}_{2} G=\bar{G} \tilde{J}_{2}$,

3. ${ }^{t} W^{t} \tilde{J}_{2} G W \in M_{4 n \times 4 n}(Z+i Z)$.

This definition is actually the quaternionic analogue of a Kähler Riemann matrix; see [24] for details. We now give a hermitian version of Theorem 7.1 from [9]. This theorem gives us examples of complex Sasakian manifolds in every possible dimension. The author is actually unaware of any other examples of compact, complex Sasakian manifolds of any dimension.

THEOREM 5.1. Let $\left\{\langle\rangle,,\left\{J_{1}, J_{2}, J_{3}\right\}\right\}$ be the standard hypercomplex structure on $\boldsymbol{C}^{2 n} \cong \boldsymbol{H}^{n}, \underline{w}=\left\{w_{1}, \ldots, w_{4 n}\right\}$ be a set of linearly independent periods in $C^{2 n}$ with period matrix $W$ and $\Gamma=\left\langle w_{1}, \ldots, w_{4 n}\right\rangle$. Let $X=C^{2 n} / \Gamma$ with hermitian projection $p:\left(C^{2 n},\langle\rangle,\right) \rightarrow(X, h)$, and let $\omega^{\prime}$ be the unique holomorphic 2-form on $X$ such that $\omega=p^{*}\left(\omega^{\prime}\right)$. Then there exists a hermitian fibration $\pi:(M, g) \rightarrow(X, h)$ with vertical fibers $\cong S^{1} \times S^{1}$ and such that $(M, g)$ is complex Sasakian, if and only if $W$ is a hyperkähler Riemann matrix.

\section{Twistor spaces and curvature equations}

It should not be mistaken that every real Sasakian manifold can somehow be "complexified" to create a complex Sasakian example. For instance, $\boldsymbol{R} P^{2 n+1}$ has 
a Sasakian structure (as described in [4]), but it's "proper" complexification is $C P^{2 n+1}$, which has no global complex contact structure, since $c_{1}\left(C P^{2 n+1}\right)>0$. In fact, $\boldsymbol{C} P^{2 n+1}$ is the twistor space of the quaternionic-Kähler manifold $\boldsymbol{H} P^{2 n+1}$.

It is worthwhile to compare these two very contrasting types of complex contact spaces: The twistor spaces of the quaternionic-Kähler manifold with nonzero scalar curvature and complex Sasakian manifolds. Both are specific cases of normal complex contact manifolds as defined in [20].

Suppose $\pi: M \rightarrow \tilde{M}$ is twistorial fibration with $(\tilde{M}, \tilde{g})$ quaternionic-Kähler with nonzero scalar curvature $\tau$. Then $M$ never has a strict complex contact structure since $c_{1}(M)$ is necessarily nondegenerate (actually positive, if $\tau>0$ ). Also, $\tilde{g}$ lifts to an Einstein associated metric $g$ on $M$ (it's also Kähler, if again $\tau$ is positive). Finally, the map $\pi$ is never holomorphic, since $\tilde{M}$ has no global complex structure.

In complete contrast, suppose that $(M, \eta, g)$ is a complex Sasakian manifold. Then, by definition, $M$ has global complex contact form. Also, $g$ is never Einstein, since, in particular, the sectional curvature of the vertical subbundle is flat, whereas the sectional curvature of a vertical vector and a horizontal vector is nonzero. Also, if $M$ is compact, then we know that it has no Kähler metrics with respect to its complex structure, since it has a global holomorphic non-closed 1 -form.

We end this paper with a discussion of how we can view the twistor space examples of complex contact manifolds with $c_{1}>0$ as those satisfying a certain associated metric curvature condition. Before we can get to this result, though, we need to describe the situation for general complex contact manifolds, which can vary in structure much more than those complex contact manifolds with a global contact form.

Let $(M, J)$ be a $2 n+1$-dimensional complex manifold with complex contact structure given by a $2 n$-dimensional holomorphic subbundle $\mathscr{H}$. Let $\{\pi=u-i v\}$ be a normalized contact structure on $M$ with resulting splitting $T M \cong \mathscr{H} \oplus \mathscr{V}$ as given by Theorem 2.1. Let $U$ and $V$ be the vertical vector fields corresponding to $u$ and $v$, respectively.

Recall that an associated metric $g$ is a hermitian metric on $M$, which satisfies the following local conditions:

1. The local real endomorphisms $G$ and $H$ defined by:

$$
\begin{aligned}
G X & =-\operatorname{skew}\left(X \mapsto \mathscr{H} \nabla_{X} U\right) \\
H X & =\operatorname{skew}\left(X \mapsto \mathscr{H} \nabla_{X} J U\right)
\end{aligned}
$$

satisfy $G^{2}=H^{2}=-i d_{\mathscr{H}}, H=G \circ J=-J \circ G$.

2. $u(X)=g(U, X), v(X)=g(V, X)$ for any vector $X$.

In [8], it is proven that an associated metric $g$ satisfies the following conditions:

1. $\mathscr{V}$ is a totally geodesic subbundle of $T M$.

2. If we set $\hat{G}(X, Y)=g(X, G Y)$ and $\hat{H}(X, Y)=g(X, H Y)$, then 


$$
\begin{aligned}
& \hat{G}=d u-\sigma \wedge v, \\
& \hat{H}=d v+\sigma \wedge u,
\end{aligned}
$$

where $\sigma(x)=g\left(\nabla_{X} U, V\right)$ is a 1 -form representing a connection on $\mathscr{V}$ as a complex line bundle over $M$. This connection is called the Ishihara-Konishi Connection with respect to $\{\pi\}$.

3. $\nabla_{X} U=-G X+h_{U} X-\sigma(X) J U, \nabla_{X} V=-H X+h_{V} X+\sigma(X) U$, for any $X \in T M$, where, for any $W \in \mathscr{V}, h_{W}$ is the real endomorphism given by

$$
h_{W}(X)=\operatorname{sym}\left(X \mapsto \mathscr{H} \nabla_{X} W\right) .
$$

4. $\nabla_{U} G=\sigma(U) H, \nabla_{J U} H=\sigma(J U) G$.

Using this terminology and machinery, we will prove the following theorem.

THEOREM 6.1. Let $M$ be a complex contact manifold with contact subbundle $\mathscr{H}$ and normalized contact structure $\{\pi=u-i v\}$. Let $T M=\mathscr{H} \oplus \mathscr{V}$ be the corresponding splitting with local vertical vector fields $\{U, V\}$ corresponding to the real forms $u$ and $v$. Suppose $g$ is an associated metric on $M$. Then $(M, g)$ is isometric to a twistor space for a quaternionic-Kähler manifold with positive scalar curvature if and only if

$$
\begin{aligned}
& R_{X Y} U=u(Y) X-u(X) Y+v(Y) J X-v(X) J Y+2 g(J X, Y) V, \\
& R_{X Y} V=-v(Y) X+v(X) Y-u(Y) J X+u(X) J Y-2 g(J X, Y) U .
\end{aligned}
$$

The proof for this theorem will actually be nothing more than pulling together various previously-known facts regarding complex contact manifolds. So, before we continue with the proof, we will now describe some of these results.

In [16], Ishihara and Konishi generated their notion of normality of complex contact manifolds. They defined local tensors

$$
\begin{aligned}
S(X, Y)= & {[G, G](X, Y)+2 \hat{G}(X, Y) U-2 \hat{H}(X, Y) V+2(v(Y) H X-v(X) H Y) } \\
& +\sigma(G Y) H X-\sigma(G X) H Y+\sigma(X) G H Y-\sigma(Y) G H X, \\
T(X, Y)= & {[H, H](X, Y)-2 \hat{G}(X, Y) U+2 \hat{H}(X, Y) V+2(u(Y) G X-u(X) G Y) } \\
& +\sigma(H X) G Y-\sigma(H Y) G X+\sigma(X) G H Y-\sigma(Y) G H X .
\end{aligned}
$$

Then they called an associated metric $g$ normal if $S=T=0$. (In this paper, we call such a metric IK-normal).

This concept was meant to be a complex analogue of normal real contact manifolds, and in some sense that is true. The most well-known complex contact manifold $C P^{2 n+1}$ is indeed IK-normal, and it is very much the complex analogue of the equally as famous Sasakian manifold $\boldsymbol{R} \boldsymbol{P}^{2 n+1}$. However, this definition of normality also leaves out the complex Heisenberg group, whose real analogue (the real Heisenberg group) is also a well-known normal real contact manifold. It is for this reason that Korkmaz in [20] expanded the definition of 
normality by calling an associated metric normal, if it only satisfied the conditions:

$$
\left.S\right|_{\mathscr{H} \wedge \mathscr{H}}=\left.S\right|_{\mathscr{H} \wedge \mathscr{V}}=\left.T\right|_{\mathscr{H} \wedge \mathscr{H}}=\left.T\right|_{\mathscr{H} \wedge \mathscr{V}}=0
$$

Returning to IK-normality, we have the following proposition from [16].

Proposition 6.2. An associated metric $g$ is IK-normal if and only if, for local $G, H, U, V$, as described above,

$$
\begin{aligned}
& \left(\nabla_{X} G\right) Y=\sigma(X) H Y-u(Y) X-v(Y) J X+g(X, Y) U+g(J X, Y) V \\
& \left(\nabla_{X} H\right) Y=-\sigma(X) G Y+u(X) J Y-v(Y) X+g(X, J Y) U+g(X, Y) V .
\end{aligned}
$$

This next proposition reworks the above conditions into those dealing with the curvature.

Proposition 6.3. An associated metric $g$ is IK-normal if and only if

$$
\begin{aligned}
& R_{X Y} U=u(Y) X-u(X) Y+v(Y) J X-v(X) J Y+2 g(J X, Y) V, \\
& R_{X Y} V=-v(Y) X+v(X) Y-u(Y) J X+u(X) J Y-2 g(J X, Y) U .
\end{aligned}
$$

Proof. It is already proven in the first part of section 4 of [16] that any IKnormal metric satisfies these curvature conditions. Conversely, we suppose that $g$ satisfies the curvature conditions. We will show that the equation of $G$ is true; the proof of the second will be completely analogous.

First, Proposition 3.1 tells us that $g$ is projectable. Thus, we have $\nabla_{X} U=$ $-G X-\sigma(X) J U$ for any vector $X$.

Direct calculation then tells us that

$$
R_{X Y} U=-\left(\nabla_{X} G\right) Y+\left(\nabla_{Y} G\right) X+2 d \sigma(X, Y) V-\sigma(Y) H X+\sigma(X) H Y .
$$

Since $\mathscr{V}$ is totally geodesic, this equation can be specialized as such:

$$
\mathscr{H} R_{J U Y} U=-\mathscr{H}\left(\nabla_{J U} G\right) Y+\sigma(J U) H Y+(J \circ \mathscr{H}) Y .
$$

Also, it is easily seen that $\mathscr{H}\left(\nabla_{J U} G\right)=\left(\nabla_{J U} G\right)$, again by the fact that $\mathscr{V}$ is totally geodesic. Comparing this result to the given curvature condition, we have that $\left(\nabla_{J U} G\right) Y=\sigma(J U) H Y$ for any $Y \in T M$.

However, we also have

$$
\begin{aligned}
\nabla_{J U} G & =\nabla_{J U}(J H) \\
& =\left(\nabla_{J U} J\right) H+J\left(\nabla_{J U} H\right) \\
& =\left(\nabla_{J U} J\right) H+\sigma(J U) H .
\end{aligned}
$$

Thus, $\nabla_{U} J=0$.

Using the Bianchi identity and a comparable expression for $\mathscr{H} R_{U Y} J U$ as above, we get that, for $X, Y \in \mathscr{H}$,

$$
g\left(R_{U V} X, Y\right)=2 g\left(\left(\nabla_{U} J\right) G X, Y\right)+2 g(J X, Y),
$$


i.e., $g\left(R_{U V} X, Y\right)=2 g(J X, Y)$ whenever $X$ and $Y$ are horizontal. From the assumed curvature condition, we also have $g\left(R_{U V} V, U\right)=2$, i.e., $g\left(R_{U V} X, Y\right)=$ $2 g(J X, Y)$ whenever $X$ and $Y$ are vertical. Thus, combining these results and using the total geodesic property of $\mathscr{V}$, we get $g\left(R_{U V} X, Y\right)=2 g(J X, Y)$ for any vectors $X$ and $Y$.

Recalling the formula $2 d \sigma(X, Y)=X \sigma(Y)-Y \sigma(X)-\sigma([X, Y])$, we also can directly calculate $d \sigma$ to get:

$$
2 d \sigma(X, Y)=g\left(R_{U V} X, Y\right)+2 g(J X, Y)
$$

i.e., $d \sigma(X, Y)=2 g(J X, Y)$ for any vectors $X$ and $Y$.

Finally, since $\hat{G}=d u-\sigma \wedge v$, we have that $d \hat{G}=-d \sigma \wedge v+\sigma \wedge \hat{H}$, i.e., in terms of the associated metric $g$,

$$
\begin{aligned}
g\left(Y,\left(\nabla_{X} G\right) Z\right)+g\left(Z,\left(\nabla_{Y} G\right) X\right)+g\left(X,\left(\nabla_{Z} G\right) Y\right) \\
=-d \sigma(X, Y) v(Z)-d \sigma(Z, X) v(Y)-d \sigma(Y, Z) v(X) \\
\quad+\sigma(X) g(Y, H Z)+\sigma(Z) g(X, H Y)+\sigma(Y) g(Z, H X)
\end{aligned}
$$

Combining this equation with those for $d \sigma$ and $R_{X Y} U$ gives the result.

Ishihara and Konishi in the same paper also prove the following important theorem.

THEOREM 6.4 (Ishihara-Konishi). If $g$ is an IK-normal associated metric on $M$, then $g$ is Kähler-Einstein and $c_{1}(M)>0$.

More recently, LeBrun in [21] proved the following theorem.

THEOREM 6.5 (LeBrun). Let $M$ be a complex contact manifold with $c_{1}(M)>0$. Then $M$ is the twistor space for a quaternionic-Kähler manifold with positive scalar curvature if and only if $M$ has a Kähler-Einstein metric.

Conversely, in the ground-breaking paper [19] of 1975, M. Konishi proved the following theorem.

THEOREM 6.6. Let $\tilde{M}$ be a quaternionic-Kähler manifold of dimension $4 m$ and positive scalar curvature. Then there exists a canonically associated $\boldsymbol{R P}^{3}$-bundle $P$ over $\tilde{M}$ admitting a Sasakian 3-structure, in such a way that $P \rightarrow \tilde{M}$ is a Riemannian submersion.

We call this bundle the Konishi bundle of the quaternionic-Kähler manifold $\tilde{M}$. Recall that we call a Riemannian manifold $(P, H)$ a 3-Sasakian manifold, if the holonomy group of the metric cone on $P$, defined by $(C(P), \bar{h})=\left(\boldsymbol{R}^{+} \times P\right.$, $\left.d r+r^{2} h\right)$, is a hyperkähler manifold. In particular, it is known that this condition is equivalent to there being three unit, Killing vector fields on $P$ $\xi_{1}, \xi_{2}, \xi_{3}$ such that: 

isfies:

1. For each $j=1,2,3$, the endomorphism $\phi_{j}$ defined by $\phi_{j}(X)=\nabla_{X} \xi_{j}$ sat-

$$
\left(\nabla_{X} \phi_{j}\right) Y=h\left(\xi_{J}, Y\right) X-h(X, Y) x i_{j} \quad \text { for all } X, Y \in T P,
$$

i.e., each $\xi_{\text {J }}$ defines a Sasakian structure on $(P, h)$,

2. $h\left(\xi_{j}, \xi_{k}\right)=\delta_{j k}$.

3. $\left[\xi_{J}, \xi_{k}\right]=2 \varepsilon_{j k l} \xi_{l}$.

See either [19] or [6] for details about 3-Sasakian manifolds. It is wellknown that at least one of the characteristic vector fields, say $\xi_{1}$, of the Konishi bundle $P$ over $\tilde{M}$, a quaternionic-Kähler manifold with positive scalar curvature, is regular, i.e., $M=P / \xi_{1}$ is a manifold. In fact, $M$ is the twistor space over $\tilde{M}$. Additionally, in [14], Ishihara and Konishi proved the following theorem.

THEOREM 6.7. Let $\left(P, h, \xi_{1}, \xi_{2}, \xi_{3}\right)$ be a 3-Sasakian manifold. If $\xi_{1}$ is regular, then the fibration $M=P / \xi_{1} \rightarrow P$ is a Riemannian submersion, and $M$ has an IK-normal complex contact structure.

Proof of Theorem 5.1. This proof now is just a matter of combining all of these results. Clearly, Proposition 5.3 and Theorem 5.7 tell us that every twistor space of a quaternionic-Kähler manifold with positive scalar curvature has an associated metric satisfying the desired curvature condition.

Conversely, if the given complex contact manifold has an associated metric satisfying the curvature condition, then this metric is IK-normal by Proposition 5.3. So, by Theorem 5.4, the metric is Kähler-Einstein, and the first Chern class of the manifold is positive. Finally, by LeBrun's result, Theorem 5.5, the complex contact manifold is the twistor space of a quaternionic-Kähler manifold with positive scalar curvature. This proves the theorem.

\section{REFERENCES}

[1] B. Aleksandrov, G. Grantcharov and S. Ivanov, Curvature properties of twistor spaces of quaternıonıc-Kähler manifolds, J. Geom., 64 (1998), 1-12.

[2] L. BÉRARD-BERgERY, Sur de nouvelles variétés riemannıenes d'Einsteın, Instıtut Elie Cartan, 6, Univ. Nancy, 1982, 1-60.

[3] A. Besse, Einsteın Manifolds, Sprınger, Berlin, 1986.

[4] D. E. Blair, Contact Manifolds in Riemannian Geometry, Lecture Notes in Math., 509, Springer, Berlin-Heıdelberg-New York, 1978.

[5] W M. Boothby and H. C. WANG, On contact manifolds, Ann. of Math., 68 (1958), $721-$ 734.

[6] C. P Boyer AND K. Galicki, The twistor space of a 3-Sasakian manifold, Internat. J. Math., 8 (1997), 31-60.

[7] M. Fernandez and A. Gray, The Iwasawa manifold, Lecture Notes in Math., 1209, Sprınger, Berlin-Heidelberg-New York, 1986, 157-159.

[8] B. ForEMAN, Variational approaches to the geometry of complex contact geometry, preprint. 
[9] B. Foreman, Boothby-Wang fibrations on complex contact manifolds, to appear in Differential Geom. Appl.

[10] B. Foreman, Twistor spaces and complex contact Hermitian geometry, in preparation.

[11] P Griffiths and J. Harris, Principles of Algebraic Geometry, Wiley, New York, 1978.

[12] Y. HatakeYama, Some notes on differentiable manifolds with almost contact structures, Tôhoku Math. J., 15 (1963), 42-48.

[13] S. IshiHARA, Quaternıon Kählerıan manifolds and fibered Riemannıan spaces with Sasakıan 3-structure, Koda1 Math. Sem. Rep., 25 (1973), 321-329.

[14] S. IshiHARA AND M. KonISHI, Real contact 3-structure and complex contact structure, Southeast Asian Bull. Math., 3 (1979), 151-161.

[15] S. Ishihara AND M. Konishi, Differential Geometry of Fibred Spaces, Publications of Study Group of Geometry, 8, Kyoto Univ., Kyoto, 1973.

[16] S. Ishihara AND M. KonISHI, Complex almost contact manifolds, Koda1 Math. J., 3 (1980), 385-396.

[17] S. IshiHARA AND M. Konishi, Complex almost contact structures in a complex contact manifold, Koda1 Math. J., 5 (1982), 30-37

[18] S. Kobayashi, Remarks on complex contact manifolds, Proc. Amer. Math. Soc., 10 (1959), 164-167

[19] M. Konishi, On manifolds with Sasakian 3-structure over quaternionic Kaehler manifolds, Koda1 Math. Sem. Rep., 26 (1975), 194-200.

[20] B. Karaman, Curvature and normality of complex contact manifolds, Thesis, Michigan State University.

[21] C. LeBrun, Fano manifolds, contact structures, and quaternıonic geometry, Internat. Math. J., 6 (1995), 419-437.

[22] S. Salamon, Quaternıonıc-Kähler manifolds, Invent. Math., 67 (1982), 143-171.

[23] R. Stong, Contact manifolds, J. Differential Geom., 9 (1974), 219-238.

[24] R. O. Wells, Differentıal Analysıs on Complex Manifolds, Sprınger, Berlin-Heıdelberg-New York, 1980.

\author{
211 Yost HaLL \\ Case Western ReSERVE University \\ Cleveland, OHio \\ USA
}

